Systematic review and meta-analysis

\section{Review: intimate partner violence is associated with termination of pregnancy}

10.1136/eb-2014-101779

Tamara L Taillieu, ${ }^{1}$ Douglas A Brownridge ${ }^{2}$

${ }^{1}$ Applied Health Sciences Program, University of Manitoba, Winnipeg, Manitoba, Canada; ${ }^{2}$ Family Social Sciences, University of Manitoba, Winnipeg, Manitoba, Canada

Correspondence to: Tamara L Taillieu, Applied Health Sciences Program, University of Manitoba, 35 Chancellor's Circle, Winnipeg, MB, Canada R3T 2N2; umtailli@myumanitoba.ca

Commentary on: Hall M, Chappell LC, Parnell BL, et al. Associations between intimate partner violence and termination of pregnancy: a systematic review and meta-analysis. PLoS Med 2014;11:e1001581.

\section{Implications for practice and research}

- Healthcare professionals need to be aware of high rates of intimate partner violence (IPV) among women seeking termination of pregnancy.

- Pregnancy termination services may be an appropriate setting for interventions aimed at reducing IPV.

- Future research must determine the magnitude of the relationship between IPV and pregnancy termination, the mechanisms through which IPV leads to pregnancy termination, and the long-term effects of IPV and pregnancy termination on women and their partners.

\section{Context}

It has been estimated that one in five women will experience some form of IPV in their lifetime. ${ }^{1}$ It has also been estimated that 42 million women undergo pregnancy termination each year, often under unsafe conditions. ${ }^{2}$ IPV as well as pregnancy termination have been recognised as global public health concerns; however, the extent to which IPV is associated with pregnancy termination remains unknown. This study aimed to review the extant literature to determine if an association exists between IPV and pregnancy termination.

\section{Methods}

Hall and colleagues performed a systematic review of the literature. Quantitative and qualitative studies published in any language were included. Studies had to be: (1) comprised of women seeking or who had undergone a termination of pregnancy where at least one aspect of IPV was also examined; (2) a randomised control trial, case-control study, cross-sectional analysis, experimental study or a secondary study with data of interest; and (3) peer reviewed. Studies were excluded if they focused on violence perpetrated by someone other than a current or former intimate partner. Seventy-four studies were included in the review, of which 23 were included in the meta-analyses examining the association between IPV, pregnancy termination and potential mediating variables.

\section{Findings}

Hall and colleagues concluded that IPV was associated with pregnancy termination. Past-year IPV prevalence rates ranged from 2.5\% to 30\% in pregnancy termination groups. Based on meta-analysis, the pooled lifetime IPV prevalence rate among women seeking or having undergone a termination of pregnancy was $24.9 \%$ (95\% CI $19.9 \%$ to 30.6\%). Significant heterogeneity existed across studies $\left(\mathrm{I}^{2}>90 \%\right)$ and was not explained by study design, study quality, sample size or country's gross national per capita income. A partner not knowing about the pregnancy termination was significantly associated with IPV (pooled OR=2.97, 95\% CI 2.39 to 3.69). From the narrative review, the authors concluded that rape, sexual assault, contraceptive sabotage and coerced decision-making were associated with termination of pregnancy and repeat pregnancy termination.

\section{Commentary}

Hall and colleagues' study was conducted in accordance with generally accepted standards. There was a clearly defined research question, a reproducible search strategy, explicitly stated inclusion and exclusion criteria, and quality assessments for each study. That said, the main findings highlighted by the review should be considered tentative at best. The lack of high-quality research in this area precludes making any strong, definitive conclusions about the association between IPV and pregnancy termination.

The past-year and lifetime prevalence rates of IPV in pregnancy termination groups suggest that an association does exist. However, these rates are remarkably similar to rates of IPV found in the general population of women ${ }^{1}$; therefore, the extent to which IPV is associated with an elevated risk of pregnancy termination remains unknown. Perhaps a more focused review could have better addressed this question. For example, including only studies with a comparison group in the meta-analysis would have allowed the authors to assess the relative risk of pregnancy termination associated with IPV.

The vast majority of quantitative studies retrieved for the review were not included in the meta-analysis to determine the prevalence of IPV among pregnancy termination groups and this needs to be recognised as an important source of bias. In addition, the substantial and unexplained heterogeneity across studies $\left(\mathrm{I}^{2}>90 \%\right)$ suggests that meta-analysis may not have been the most appropriate method to address the research question. ${ }^{3}$

While there appears to be an association between IPV and termination of pregnancy, the extent to which the risk of pregnancy termination is elevated among women experiencing IPV remains unknown. Future research aimed at addressing gaps in the literature identified by Hall and colleagues is needed in order to better understand the relationship between IPV and termination of pregnancy.

\section{Competing interests None.}
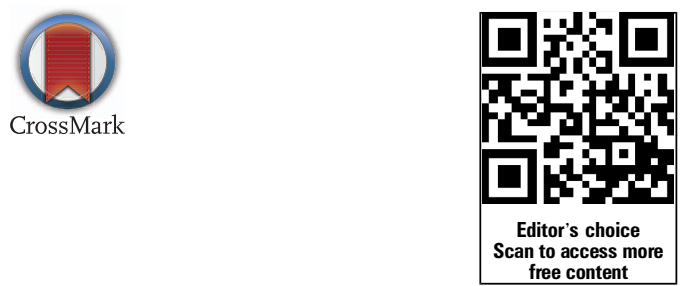

\section{References}

1. World Health Organization. Addressing violence against women and achieving the Millennium Development Goals. Geneva, Switzerland: World Health Organization, 2005.

2. World Health Organization. Unsafe abortion: global and regional estimates of the incidence of unsafe abortion and associated mortality in 2008. 6th edn. Geneva, Switzerland: World Health Organization, 2011.

3. Noordzij M, Hooft L, Dekker FW, et al. Systematic reviews and meta-analyses: when they are useful and when to be careful. Kidney Int 2009;76:1130-6. 